\title{
La Comprensión Narrativa en Edad Preescolar: Un Instrumento para su Medición
}

\section{Narrative Comprehension in the Preschool Age: An Assessment Instrument}

\author{
Katherine Strasser \\ Pontificia Universidad Católica de Chile \\ Antonia Larraín \\ Universidad Alberto Hurtado \\ Soledad López de Lérida y María Rosa Lissi \\ Pontificia Universidad Católica de Chile
}

\begin{abstract}
La comprensión narrativa es una habilidad fundamental en la alfabetización infantil, poco atendida por investigadores y educadores por las dificultades para comprenderla y medirla, sobre todo a edades tempranas. Se reportan resultados de un estudio cuyo objetivo fue elaborar y validar un instrumento para la medición de la comprensión narrativa en niños en edad preescolar. Participaron 117 niños y niñas chilenos entre 3 años 6 meses y 5 años 1 mes de edad, pertenecientes a jardines infantiles de la Región Metropolitana. Se encontró evidencia que apoya la confiabilidad y la validez concurrente del instrumento, de fácil y rápida aplicación y codificación. Se discuten las implicancias de contar con un instrumento para medir comprensión narrativa que sea adecuado a la población preescolar chilena, así como estudios futuros necesarios para mejorar el instrumento.
\end{abstract}

Palabras clave: comprensión narrativa, preescolar, propiedades métricas, comprensión lectora, instrumento de medición.

Narrative comprehension is a fundamental ability for children's literacy development that has been largely ignored by researchers and educators, due to the difficulties in understanding and measuring it, particularly in the early ages. The results of a study, whose goal was to construct and validate an instrument to measure narrative comprehension in preschool age children, are presented. Participants were 117 Chilean children aged 3 years and 6 months to 5 years and 1 month. Evidence was found to support the reliability and concurrent validity of the instrument, which is simple and quick to administer and code. The implications of having an instrument to measure narrative comprehension that is adequate for the Chilean preschool population are discussed, as are future studies to improve the instrument.

Keywords: narrative comprehension, preschool, metric properties, reading comprehension, assessment instrument.

\section{Introducción}

El paradigma de la alfabetización emergente (Teale \& Sulzby, 1986; Whitehurst \& Lonigan, 1998) sugiere que las habilidades de alfabetización se desarrollan desde muy temprana edad, previo a las primeras experiencias escolares. Entre estas habilidades de alfabetización emergente, las que han sido atendidas más ampliamente son

Katherine Strasser, Escuela de Psicología, Pontificia Universidad Católica de Chile, Santiago, Chile.

Antonia Larraín, Escuela de Psicología, Facultad de Psicología, Universidad Alberto Hurtado, Santiago, Chile.

Soledad López de Lérida, Escuela de Psicología, Pontificia Universidad Católica de Chile, Santiago, Chile.

María Rosa Lissi, Escuela de Psicología, Pontificia Universidad Católica de Chile, Santiago, Chile.

La correspondencia relativa a este artículo debe ser dirigida a Katherine Strasser, Escuela de Psicología, Pontificia Universidad Católica de Chile, Avenida Vicuña Mackenna 4860, Macul, Santiago, Chile. E-mail: kstrasse@uc.cl

Este estudio fue posible gracias al financiamiento del Fondo Nacional de Ciencia y Tecnología de Chile (Proyecto FONDECYT N 1080241). 
el reconocimiento de letras, la conciencia fonológica y el vocabulario (Van Kleeck, 2008), mientras que la comprensión de historias -o comprensión narrativa- ha sufrido una notoria falta de atención (Kendeou, Lynch, van den Broek, Espin, White \& Kremer, 2005; Lynch, van den Broek, Kremer, Kendeou, White \& Lorch, 2008; Paris \& Paris, 2003; Strasser \& Lissi, 2009). Sin embargo, diversas investigaciones señalan que la comprensión narrativa en la etapa preescolar se relaciona con la comprensión lectora en la etapa escolar (Kendeou et al., 2005; Paris \& Paris, 2003), aun luego de controlar el efecto de otras variables, como el vocabulario (Kim, Kendeou, van den Broek, White \& Kremer, 2008; Paris \& Paris, 2003; van den Broeck et al., 2005).

¿Por qué ha sido tan ignorada esta habilidad en la educación preescolar? Entre otras causas, la falta de claridad sobre su naturaleza y la forma más adecuada de evaluarla han contribuido a su ausencia del curriculum preescolar (van den Broek et al., 2005). Sin embargo, la relación directa entre comprensión narrativa en la edad preescolar y comprensión lectora posterior (Kim et al., 2008; Paris \& Paris, 2003; van den Broeck et al., 2005) releva la atención que debe ponerse al desarrollo de la comprensión narrativa en la edad preescolar.

El objetivo de este estudio fue elaborar un instrumento para evaluar la comprensión narrativa en preescolares. Para ello discutiremos, en primer lugar, la naturaleza del constructo a ser medido, así como su relación con otros constructos similares, como la comprensión lectora y auditiva.

\section{¿Qué Es la Comprensión Narrativa?}

En primer lugar, es conveniente aclarar que el concepto de comprensión narrativa se refiere a la comprensión de historias sin especificar la forma en que son presentadas. Cuando las personas acceden a las historias a través de la lectura, hablamos de comprensión lectora y cuando las historias son escuchadas, hablamos de comprensión auditiva. Tanto la comprensión lectora como la auditiva pueden referirse a distintos textos, incluyendo historias, textos expositivos e instrucciones, pero en este artículo, al utilizar los términos comprensión lectora y auditiva, nos referimos específicamente a la comprensión lectora y auditiva de historias.

Van den Broek et al. (2005) plantean que la comprensión de un texto, más que un fenómeno unitario, es un conjunto de habilidades y destrezas que involucra: a) la interpretación de información literal, b) el uso de conocimiento previo y c) la construcción de una representación coherente y significativa del texto.

Aunque la información literal no es suficiente para establecer relaciones significativas entre distintos elementos de una historia, ella es indispensable. Recuperar e interpretar la información literal es el primer paso para comprender una narración. Por ello, tanto el vocabulario como el conocimiento previo están ligados a la comprensión de una historia (Cain, Oakhill, Barnes \& Bryant, 2001; Cain, Oakhill \& Bryant, 2004; Kendeou, Rapp \& van den Broek, 2003; Lynch et al., 2008). Por ejemplo, las primeras líneas del cuento de Fernando Krahn (2006, p. 3), Huellas Gigantes, dicen así:

Una fría mañana de domingo.... Mamá

Úrsula había puesto al horno un pan de cereales cuyo delicioso olor levantó con entusiasmo al resto de la familia.

Para interpretar esta información es relevante saber que el pan de cereal se come al desayuno, que se cocina en el horno, que si está recién cocinado estará calentito y que esto es bueno, y que un aroma agradable tiene el potencial de "levantar" a una persona dormida o aletargada. Para interpretar el párrafo hay que recuperar de la memoria estos conocimientos previos e integrarlos con la información literal.

Pero el conocimiento previo y el recuerdo de información literal no son suficientes para explicar la comprensión narrativa (Cain et al., 2001, 2004). También es fundamental inferir información que no está explícita en el texto ni es parte del conocimiento previo (van den Broek et al., 2005; Van Kleeck, 2008; Van Kleeck, Vander Woude \& Hammett, 2006). Tómese por ejemplo, el siguiente trozo del libro ¿Eres tú mi mamá? de Eastman (1960, pp. 6-7):

$Y$ del huevo salió un pajarito.

- ¿Dónde está mi mamá? - preguntó.

La buscó. Miró para arriba, no la vio. 
En este trozo nos parece evidente que el pajarito miró hacia arriba para buscar a su mamá. Sin embargo, esta conexión no es explícita en el texto; es necesario inferirla. Los elementos de una historia deben ser articulados por medio de relaciones de coherencia, las que en su mayor parte -como en el ejemploson inferidas, no explícitas. Sin estas relaciones, la historia es una serie de eventos separados sin relación entre sí (Lynch et al., 2008; Makdissi \& Boisclair, 2006; van den Broek et al., 2005; Van Kleeck et al., 2006). La evidencia sugiere que la capacidad de inferir relaciones de coherencia está relacionada con la comprensión de historias, más allá del conocimiento previo y la memoria de trabajo (Cain et al., 2004; Van Kleeck, 2008; Van Kleeck et al., 2006). Entre las relaciones de coherencia, las conexiones causales son las más importantes para la comprensión (Cain, 2003; Kim et al., 2008; Lynch et al., 2008). A diferencia de las inferencias literales, donde se extrae información descriptiva necesaria para entender el nivel textual, las inferencias causales implican la reconstrucción de las conexiones que vinculan los eventos de la historia; entiéndase: el problema desencadenante, las intenciones y sentimientos de los personajes, las acciones y sus consecuencias, los problemas y las soluciones.

Ciertos elementos de las historias tienen un potencial privilegiado para dar coherencia. Las intenciones y motivaciones son esenciales para comprender una historia con personajes animados, pues dan sentido a sus acciones (Lynch \& van den Broek, 2007). De hecho, la sensibilidad de los niños y niñas a estos elementos psicológicos tiene relación directa con su comprensión narrativa (Cain, 2003; Makdissi \& Boisclair, 2006; Trabasso \& Wiley, 2005; Wenner, 2004).

Podemos decir, entonces, que la comprensión narrativa es un tipo de pensamiento verbal que se moviliza para construir una organización significativa y sinóptica de una historia. Involucra la integración de la información literal y el conocimiento previo, a través de inferencias, entre las cuales destacan las inferencias causales, particularmente, las inferencias sobre los estados psicológicos de los personajes. El resultado de estos procesos es la organización cohe- rente de los elementos de la narración.

Una definición clara de este constructo aumenta la probabilidad de que los currículos de educación preescolar incluyan actividades orientadas a su desarrollo. Pero los educadores no solo necesitan directrices sobre cómo estimular determinadas habilidades sino, también, instrumentos para evaluarlas con el objetivo de mejorar su instrucción (Snow, Griffin \& Burns, 2005). Existen varias dificultades para medir habilidades de comprensión narrativa a temprana edad y hay muy pocos instrumentos adecuados, en especial en el idioma español. Es urgente, por tanto, desarrollar instrumentos que permitan medir estas habilidades en esta etapa.

\section{Medición Temprana de la Comprensión Narrativa}

La manera tradicional de medir la comprensión de historias en la edad escolar es entregar un texto para que los estudiantes, luego de leerlo, respondan preguntas. Evidentemente, esto no es útil en preescolares, quienes no leen con fluidez. Otro enfoque es hacer preguntas sobre historias contadas por un adulto. Sin embargo, esta medida descansa fuertemente en la atención, la memoria de corto plazo y el vocabulario. Algunos estudios han medido la comprensión narrativa a través del pensamiento hablado de los participantes en el momento en que escuchan historias (thinking out loud) para evaluar la realización de inferencias on-line (Lynch \& van den Broek, 2007). Un cuarto enfoque consiste en evaluar la calidad de las narrativas producidas por los preescolares en respuesta a una pregunta o en base a un estímulo visual, como una lámina (Peterson \& McCabe, 2004). Un problema importante de este tipo de mediciones es la dificultad de obtener muestras de lenguaje suficientemente largas para ser representativas del lenguaje del niño o niña (Lee \& Canter, 1971).

Recientemente un equipo de investigación ha desarrollado una forma de medir la comprensión narrativa de niños y niñas de seis años, a los que se les presentan programas de televisión para luego hacer que cuenten lo que sucedió o respondan pre- 
guntas (Lynch et al., 2008; Kendeou et al., 2005; Kim et al., 2008; van den Broek et al., 2005). Los estudios muestran que esta forma de medir es equivalente a la de las historias que son relatadas oralmente (comprensión auditiva). Sin embargo, esta forma de medición también descansa fuertemente sobre la atención, la memoria de corto plazo y el vocabulario.

Paris y Paris (2003) desarrollaron un instrumento para medir comprensión narrativa en niños y niñas de cinco a ocho años que controla en parte el efecto del vocabulario y la memoria. Usando libros mudos (sin palabras) les pedían a los participantes que recorrieran el libro comentándolo, luego que relataran la historia sin mirar el libro (recuento sin libro) y, por último, que respondieran preguntas. Para evaluar la confiabilidad y la validez concurrente de este instrumento, Paris y Paris (2001, 2003) condujeron dos estudios con 158 y 91 niños y niñas de kindergarten, primero y segundo grado de primaria, respectivamente. La confiabilidad inter-codificador fue estimada por medio del porcentaje de acuerdo de dos codificadores que evaluaron un $30 \%$ de los casos en cada estudio, el que fue mayor al $90 \%$ para todas las preguntas, pero no se consignó la consistencia interna de la prueba. La prueba arrojó correlaciones directas con recuento de historias escuchadas y con una prueba de conceptos sobre el texto escrito. En un tercer estudio $(N=141)$ los autores evaluaron la validez predictiva $\mathrm{y}$ encontraron que el instrumento tenía una relación directa con la comprensión lectora un año más tarde.

Entre otras, las ventajas de utilizar un libro mudo son que: a) no depende de habilidades de decodificación ni es demasiado dependiente del vocabulario del niño; b) disminuye la carga de la memoria porque los participantes contestan observando las ilustraciones del libro; c) es una medición sencilla y de bajo costo y d) permite medir la realización de inferencias. Sin embargo, Paris y Paris $(2001,2003)$ plantean que los estudios realizados por ellos dejan abiertas varias preguntas, entre las cuales destacamos dos de interés para este estudio: a) ¿puede usarse en niños y niñas menores de cinco años, cuyas habilidades léxicas y sintácticas difieren considerablemente de las de niños y niñas mayores? y b) ¿es adecuado para otras poblaciones diferentes a la estudiada?

Para evaluar la adecuación de este instrumento en una población hispano-parlante y de menor edad, Strasser y sus colaboradoras lo aplicaron a un grupo de participantes chilenos de kindergarten (Strasser \& Lissi, 2006) y a otro de tres años (Strasser, Lissi \& Larraín, 2009). Para ello utilizaron el libro Frog, where are you? de Mercer Mayer (1994) ${ }^{1}$ y el protocolo original de Paris y Paris, que consta de 10 preguntas que reciben 0,1 ó 2 puntos. Al aplicar el instrumento a niños y niñas de kindergarten, Strasser y Lissi (2006) no quedaron satisfechas con sus características psicométricas. Trabajando con tres codificadoras, se codificaron dos veces las 113 narrativas, encontrando que solo cuatro de los 10 coeficientes Kappa de Cohen alcanzaron un valor igual o superior a 0,7 $(p<0,005)$, mientras que dos estuvieron en el rango entre 0,4 y $0,5(p<0,05)$ y los otros cuatro no fueron significativos. El alfa de Cronbach para la escala de 10 preguntas luego de reconciliar los puntajes definitivos fue de solo 0,67. En un segundo intento se capacitó a otras tres codificadoras que realizaron todo el proceso nuevamente y los resultados fueron incluso más bajos (Strasser \& Lissi, 2006). El intento de aplicar la tarea a 16 niños y niñas menores de cuatro años mostró que estos ni siquiera podían responder a las preguntas acerca de este libro, el que pareció ser muy complejo para ellos (Strasser, Lissi \& Larraín, 2009).

Con el propósito de elaborar un instrumento para la medición de la comprensión narrativa adecuado y pertinente para la realidad de la población chilena preescolar, realizamos un estudio que evaluó la validez concurrente, la confiabilidad inter-jueces y la consistencia interna de una tarea de comprensión narrativa en base a un nuevo libro sin palabras, más sencillo que el utilizado por Paris y Paris (2003) y con preguntas más específicas, con el propósito de

\footnotetext{
1 Aunque el título del libro es en inglés, el libro no tiene texto, por lo cual puede ser y ha sido utilizado en estudios en diversos idiomas (Berman \& Slobin, 1994).
} 
mejorar el acuerdo entre los codificadores. Este estudio representa una extensión del trabajo realizado por Paris y Paris (2003) en la medición de comprensión narrativa mediante libros sin palabras a niños y niñas pequeños hablantes de español.

Relación de la Comprensión Narrativa con Otros Constructos Lingüísticos en la Etapa Preescolar

La prueba que reportamos en este artículo se denominó Prueba de Comprensión Narrativa Preescolar (en adelante PCNP). Para evaluar su validez concurrente, conviene conocer qué otras habilidades de lenguaje tienen relación con la comprensión narrativa, con el objetivo de determinar si el instrumento captura estas relaciones. El vocabulario receptivo (VR) se usa ampliamente como una medida general de la habilidad verbal en preescolares, en parte porque su medición no requiere de una respuesta verbal y, por ende, soslaya problemas como la excesiva timidez o un lento desarrollo del lenguaje expresivo. Así, nuestra primera predicción es que la PCNP tendrá una correlación directa con una prueba de $V R$, puesto que ambas pruebas miden aspectos de la habilidad verbal. Sin embargo, el rol causal del VR no es tan importante para la comprensión narrativa de un cuento mudo como lo es cuando escuchamos o leemos un texto con palabras específicas que podemos desconocer (Cromley \& Azevedo, 2007; Elleman, Lindo, Morphy \& Compton, 2009; Ouellette, 2006; Stahl \& Fairbanks, 1986). Una segunda predicción, entonces, es que la correlación entre la PCNP y el $V R$ será menor que la correlación entre VR y una prueba de comprensión en base a un texto específico. Debido a que se trata de prelectores, no podemos utilizar para estos efectos una prueba de comprensión lectora, pero sí una prueba de comprensión auditiva de cuentos (CAC).

Una tercera predicción es que la correlación entre la PCNP y la CAC será significativa y directa, puesto que se requieren habilidades comunes para comprender un libro mudo y una historia escuchada. Nuestra última predicción es que la correlación entre la PCNP y la CAC será mayor que la de la PCNP y el VR, puesto que tanto el rendimiento en la CAC como en la PCNP estarían influidos no solo por el VR sino, también, por otros factores, como la experiencia con historias (Peterson \& McCabe, 2004; Strasser \& Lissi, 2006) y la teoría de la mente (Happé, 1994; Makdissi \& Boisclair, 2006). De esta forma, las correlaciones esperadas respecto de las que se evaluará la validez concurrente del instrumento, son como sigue:

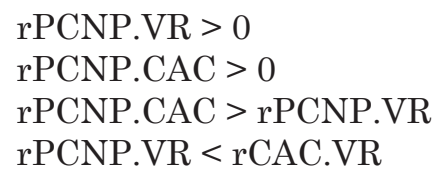

Método

\section{Participantes}

Los participantes pertenecían a tres jardines infantiles de la comuna de La Florida en Santiago de Chile, en el invierno del año 2008. Los jardines fueron seleccionados por conveniencia, debido a su cercanía con la Universidad desde donde se dirigió el estudio y por atender a la población de interés (niños y niñas de familias de nivel socioeconómico [NSE] bajo y medio-bajo). Todos los niños y niñas entre $3 \mathrm{y}$ medio y 5 años de edad que asistían a los jardines $(N=171)$ eran elegibles para el estudio, pero 22 de ellos no pudieron ser contactados a la entrada o salida del jardín, ya que no eran los padres quienes los llevaban o recogían. De los 149 padres o madres que fueron contactados, 117 dieron su consentimiento para participar en el estudio. Los 32 restantes no negaron su consentimiento pero no devolvieron las cartas a los jardines. Una prueba $t$ para muestras independientes mostró que la edad de los niños y niñas con y sin consentimiento firmado no era significativamente diferente, $t(147)=$ $0,71, p=0,477$. La prueba de Chi cuadrado mostró que la distribución por sexo tampoco era significativamente diferente entre los niños con y $\sin$ consentimiento, $\chi^{2}(1, N=$ $149)=0,149, p=0,699$. Cincuenta y cuatro de los 117 participantes eran mujeres. Los niños y niñas tenían entre 3 años y 6 meses y 5 años y 1 mes de edad, y la edad promedio fue de 4 años y 3 meses al inicio 
del estudio $(D E=5,6)$. Nueve participantes asistían paralelamente a una Escuela de Lenguaje por haber sido diagnosticados con dislalias, pero sin otros trastornos del lenguaje. No controlamos este factor, pues los trastornos de articulación no asociados a trastornos de vocabulario o sintaxis no se relacionan con la comprensión de historias (Peterson, Pennington, Shriberg \& Boada, 2009; Raitano, Pennington, Tunick, Boada \& Shriberg, 2004). Todos los participantes eran prelectores.

\section{Instrumentos}

Prueba de Comprensión Narrativa Preescolar (PCNP). La prueba consiste en un libro comercial y un cuestionario con una pauta de codificación. Escogimos un libro sin palabras más simple que el usado por Paris y Paris (2003), para facilitar la comprensión de niños más pequeños. El libro es Chigüiro encuentra ayuda de Ivar Da Coll (1987), que consta de 18 páginas con ilustraciones en colores, las que narran la historia de un capibara (o carpincho) que trata de alcanzar unas bananas. El libro posee una estructura narrativa canónica, con varios problemas y una resolución. Las ilustraciones reflejan las intenciones, problemas y emociones del personaje, por lo que se presta para evaluar la capacidad de comprensión de los elementos que dan coherencia a la historia. A su vez, el libro es breve y simple. El cuestionario consta de 19 preguntas. La mayoría son preguntas de inferencia causal acerca de intenciones, pensamientos, sentimientos, problemas, diálogo y resolución. También contiene una pregunta de información literal (personajes) y una que mide la comprensión del tema general de la historia (título). El procedimiento es similar al de Paris y Paris (2003), pero el recuento sin libro fue eliminado por ser poco relevante en los estudios originales. La tarea quedó formada por tres partes: introducción, visión preliminar y preguntas. En la introducción se muestra el libro y se explica que no tiene ni letras ni palabras, sino que los dibujos cuentan la historia. Durante la visión preliminar se le indica al niño o niña que mire todas las páginas y que vaya diciendo en voz alta todo lo que se le ocurra al ver las ilustraciones. El objetivo de esta parte no es evaluar, sino que los participantes construyan una representación de la historia para que luego puedan responder a las preguntas. En la tercera parte de la tarea se formulan las 19 preguntas del test, mostrando las páginas del libro relevantes a cada pregunta. Las respuestas se anotan literalmente. Cada respuesta recibe 0,1 ó 2 puntos según su relevancia y presencia de una inferencia de coherencia. El rango teórico de puntaje de la prueba es de 0 a 38 puntos. Las características psicométricas de la prueba se consignan en la sección de resultados.

Vocabulario receptivo. Utilizamos el Test de Vocabulario en Imágenes de Peabody (TVIP), en el que los participantes deben señalar la imagen correspondiente a una palabra de entre cuatro alternativas. Este instrumento estadounidense fue normado con 1.219 niños y adolescentes monolingües hispanoparlantes procedentes de México y 1.488 niños y niñas de Puerto Rico (Dunn, Padilla, Lugo \& Dunn, 1986). La consistencia interna de la prueba (split-half) fue de 0,93 (correlación media corregida por la fórmula de Spearman-Brown). La validez concurrente fue evaluada con respecto a la versión en español de la escala de Kaufman-ABC, una prueba de desarrollo cognitivo (Gómez-Palacio, Rangel $\&$ Padilla, 1985). Las correlaciones con esta prueba estuvieron entre 0,25 y 0,59 (puntaje global) y entre 0,28 y 0,69 (escala de rendimiento) (Dunn et al., 1986). El TVIP no tiene normas para la población chilena, pero lo consideramos adecuado para el estudio puesto que nos interesaba la variabilidad entre los participantes y no requeríamos hacer juicios clínicos sobre el desarrollo del vocabulario de estos ${ }^{2}$.

Comprensión auditiva de cuentos. Esta prueba fue especialmente desarrollada en este estudio para evaluar la comprensión de tres cuentos. La prueba consiste en un

\footnotetext{
2 El único instrumento de VR con normas chilenas fue estandarizado en una sola región del país con solo 120 niños divididos en 12 rangos de edad (Echeverría, Herrera \& Vega, 1993). Además, a nuestro juicio, el instrumento tiene problemas en cuanto a la claridad de las ilustraciones.
} 
cuestionario de 39 preguntas, 13 para cada cuento. Los participantes escucharon tres cuentos cuatro veces cada uno en el transcurso de tres semanas y se les aplicó el cuestionario entre dos y ocho días luego de la última lectura. El cuestionario incluye preguntas de recuerdo de información literal y de inferencia. Las examinadoras anotaron todas las respuestas y estas fueron codificadas por personas previamente capacitadas. Cada pregunta recibe un puntaje de 0, 1 ó 2 puntos, resultando teóricamente una escala de 0 a 78 puntos. La confiabilidad intercodificador de la prueba fue estimada por medio del coeficiente Kappa de Cohen entre dos codificadoras independientes que codificaron 50 casos. Tres de las 39 preguntas obtuvieron coeficientes entre 0,4 y 0,5 ; cinco, entre 0,5 y 0,69 y el resto, sobre 0,7 (todos significativos con $p<0,001)$. La consistencia interna de la prueba (alfa de Cronbach) fue de 0,898 .

\section{Procedimiento}

Este estudio estuvo inserto dentro de un estudio mayor de carácter experimental, que incluía la asignación aleatoria de los participantes a un grupo experimental y a un grupo control. El grupo experimental del estudio principal recibió una intervención consistente en lecturas repetidas de tres cuentos, y todos los participantes fueron evaluados antes y después de la intervención del estudio principal. Las pruebas incluidas en el presente estudio fueron administradas en su mayoría antes de la intervención, con algunas excepciones que se detallan cuando corresponde.

Las directoras de los jardines infantiles fueron contactadas por teléfono para participar en el estudio principal. Posteriormente se realizó una reunión con la directora de cada jardín y las profesoras de los niveles involucrados, para aclarar procedimientos, incluyendo la identificación de los horarios menos disruptivos y la forma de devolver la información a los jardines al concluir el estudio.

Los padres o madres fueron contactados a la hora de entrada o salida de los jardines infantiles, momento en que se les explicó el estudio. Los padres que aceptaron participar firmaron una carta de consentimiento, mientras que algunos prefirieron discutir el tema con su cónyuge y enviar la carta posteriormente. El asentimiento de los niños y niñas fue solicitado al retirarlos de la sala, diciendo: "Vamos a mirar unos dibujos y leer unos cuentos, ¿quieres venir conmigo?" Si el niño o niña no quería salir de la sala, no se le insistía y se le preguntaba en otra ocasión. Ante un segundo rechazo, las examinadoras no debían volver a contactar al niño o niña, pero ninguno rechazó por segunda vez su participación. Al retirar a los niños y niñas de la sala era frecuente que otros compañeros pidieran salir, ante lo cual las examinadoras debían decir: "Ahora voy a trabajar con NN. A lo mejor otro día te toca a ti." Si un niño o niña no tenía consentimiento, se le indicaba que no se trabajaría con él o ella porque no estaba en la lista.

Los instrumentos de evaluación fueron administrados individualmente en una sala aparte por estudiantes de psicología. Las pruebas fueron codificadas por ayudantes distintas a las que las administraron.

Los instrumentos fueron administrados en el mismo orden a todos los participantes, descartando así efectos diferenciales.

La prueba de VR y la primera aplicación de la PCNP se administraron antes de la intervención del estudio principal y, por lo tanto, no estuvieron expuestas a sus efectos. Se aplicó primero la Prueba de VR y aproximadamente una semana más tarde, la PCNP, pues no existe ninguna evidencia ni razón teórica para la suposición de que la prueba de VR usada tenga efectos sobre la comprensión de historias.

La prueba de CAC se aplicó luego de la intervención, pues su administración requería que los participantes fueran expuestos a la lectura de tres cuentos, por lo que cualquier efecto de la intervención es inherente al instrumento y no está sistemáticamente asociado con ninguna característica de los participantes. Estas lecturas se realizaron en una sala aparte a grupos de tres a seis participantes. La prueba se aplicó a una submuestra de 72 niños seleccionados aleatoriamente, aproximadamente tres semanas más tarde que la primera aplicación de la PCNP. Tampoco existe razón teórica por la cual la PCNP pueda tener efectos sobre la comprensión auditiva. 
Una segunda aplicación de la PCNP se realizó a la totalidad del grupo control del estudio principal $(N=32)$ y a una submuestra del grupo experimental seleccionada aleatoriamente $(N=38)$, aproximadamente tres meses después de la primera (el promedio de días fue de 82 , con un rango de 71 a 105 días). Esta administración se efectuó después de la intervención, por lo cual pudo haber habido efectos de la misma. Discutiremos estos en la sección de Resultados.

Los cuestionarios fueron almacenados bajo llave en la Pontificia Universidad Católica de Chile. Los datos digitalizados no fueron encriptados, pero solo las investigadoras tuvieron acceso a ellos. Al finalizar el estudio se realizó un seminario de entrega de resultados con directoras y profesoras. En este seminario se entregaron resultados sobre la efectividad de las estrategias de lectura y no sobre el desempeño de niños, cursos o jardines individuales.

\section{Análisis de Datos}

Para codificar la PCNP desarrollamos un manual de codificación para asignar a cada pregunta un puntaje 0,1 ó 2 , según si existía una inferencia y si esta era suficiente para comprender los eventos de la página en relación a la historia. Capacitamos a dos codificadoras en una sesión de dos horas que incluyó ejemplos y discusiones. Los casos utilizados en la capacitación fueron codificados nuevamente al finalizar el proceso de codificación.

Estimamos la confiabilidad inter-codificador mediante el coeficiente Kappa de Cohen. Las dos codificadoras codificaron $54 \%$ de los casos $(n=63)$ en tres rondas sucesivas de 21 casos cada una. Al finalizar cada ronda, se calculó el acuerdo y se aclararon criterios. Cuando hubo desacuerdos, el puntaje final se decidió por medio de discusión entre las codificadoras. El resto de los casos fueron codificados por una sola codificadora.

Para estimar la sensibilidad de la PCNP al cambio evolutivo calculamos el coeficiente de correlación lineal de Pearson entre el puntaje y la edad. También estimamos la sensibilidad al cambio, utilizando la prueba $t$ de Student para muestras dependientes, con el propósito de determinar si el puntaje de los niños y niñas que hicieron la prueba dos veces aumentó significativamente.

Para estimar la validez concurrente de la PCNP examinamos sus correlaciones $(r$ de Pearson) con las pruebas de VR y CAC. Para comparar estas correlaciones entre sí utilizamos $\mathrm{Z}$ de Fisher, con el fin de determinar si el patrón de correlaciones era similar al predicho.

Para evaluar las contribuciones relativas de VR y CAC a los puntajes de la PCNP realizamos un análisis de regresión múltiple con PCNP como variable dependiente y VR y CAC como predictores.

\section{Resultados}

A continuación presentamos los resultados de la aplicación del instrumento. En primer lugar, presentamos los resultados descriptivos, incluyendo la dificultad de cada ítem y los estadísticos descriptivos del instrumento total y de las otras variables del estudio. En segundo lugar, presentamos los resultados relacionados con la confiabilidad del instrumento (acuerdo entre codificadores y consistencia interna). En tercer lugar, mostramos la evidencia relacionada con la sensibilidad de la prueba al cambio evolutivo. Finalmente, presentamos los resultados relacionados con la validez concurrente de la prueba.

\section{Resultados Descriptivos}

En la Tabla 1 hemos resumido los estadísticos descriptivos de las tres variables del estudio en los distintos momentos. El puntaje promedio en la PCNP muestra que los participantes son capaces de responder preguntas de comprensión narrativa, incluyendo preguntas de inferencia causal.

En la Tabla 2 se puede observar el porcentaje de participantes que obtuvo 0,1 ó 2 puntos en cada pregunta. En la mayoría de ellas un porcentaje considerable alcanza la puntuación máxima. Las preguntas por pensamientos, sentimientos e intenciones de los personajes no son las más difíciles: en nueve de las diez preguntas de este tipo, un tercio o más de los niños obtiene el puntaje máximo. 
Tabla 1

Estadísticos Descriptivos de las Variables del Estudio

\begin{tabular}{lrrrrr}
\hline Variable & \multicolumn{1}{c}{$N$} & Min & Max & Promedio & \multicolumn{1}{c}{$D E$} \\
\hline PCNP primera administración & 117 & 0 & 35 & 20,82 & 7,83 \\
PCNP segunda administración & 70 & 15 & 36 & 25,86 & 5,62 \\
Comprensión Auditiva de Cuentos & 72 & 14 & 67 & 44,65 & 13,64 \\
Vocabulario receptivo (estándar) & 117 & 74 & 143 & 106,6 & 16,23 \\
\hline
\end{tabular}

Tabla 2

Porcentaje de Niños y Niñas que Obtuvieron Puntajes 0, 1 ó 2 en Cada Pregunta de la PCNP

\begin{tabular}{|c|c|c|c|c|c|}
\hline \multirow{2}{*}{ Pregunta } & \multicolumn{3}{|c|}{ Puntaje } & \multirow{2}{*}{ Promedio } & \multirow{2}{*}{$D E$} \\
\hline & 0 & 1 & 2 & & \\
\hline 1. ¿Quiénes estaban en el cuento? & 4,1 & 5,7 & 86,1 & 1,85 & 0,46 \\
\hline 2. ¿Qué está pensando Chigüiro aquí? & 17,2 & 20,5 & 58,2 & 1,43 & 0,78 \\
\hline 3. ¿Qué va a hacer Chigüiro aquí? & 23,8 & 20,5 & 51,6 & 1,29 & 0,84 \\
\hline 4. ¿Para qué va a hacer eso? & 16,4 & 14,8 & 64,8 & 1,50 & 0,77 \\
\hline 5. ¿Qué pasó aquí? & 25,4 & 33,6 & 36,9 & 1,12 & 0,80 \\
\hline 6. ¿Cómo se siente Chigüiro aquí? & 33,6 & 15,6 & 46,7 & 1,14 & 0,91 \\
\hline 7. ¿Por qué se siente así? & 47,5 & 6,6 & 41,8 & 0,94 & 0,97 \\
\hline 8. ¿Qué quiere hacer Chigüiro aquí? & 29,5 & 21,3 & 45,1 & 1,16 & 0,87 \\
\hline 9. ¿Para qué quiere hacer eso? & 19,7 & 11,5 & 64,8 & 1,47 & 0,82 \\
\hline 10. ¿Qué está pensando Chigüiro aquí? & 33,6 & 48,4 & 13,9 & 0,79 & 0,68 \\
\hline 11. ¿Qué le está diciendo Mono a Chigüiro aquí? & 60,7 & 32,0 & 3,3 & 0,40 & 0,56 \\
\hline 12. ¿Qué está diciendo Chigüiro aquí? & 36,1 & 14,8 & 45,1 & 1,09 & 0,92 \\
\hline 13. ¿Qué pasó aquí? & 20,5 & 17,2 & 58,2 & 1,39 & 0,82 \\
\hline 14. ¿Por qué pasó eso? & 29,5 & 19,7 & 46,7 & 1,18 & 0,88 \\
\hline 15. ¿Cómo se sienten Chigüiro y Mono aquí? & 36,9 & 27,9 & 31,1 & 0,94 & 0,84 \\
\hline 16. ¿Por qué se sienten así? & 45,9 & 13,1 & 36,9 & 0,91 & 0,93 \\
\hline 17. Si Chigüiro se encuentra otros plátanos ¿Qué debería hacer? & 68,0 & 8,2 & 19,7 & 0,50 & 0,82 \\
\hline 18. ¿Qué pasó en el cuento? & 36,9 & 41,0 & 18,0 & 0,80 & 0,73 \\
\hline 19. ¿Cómo crees que se llama este cuento? & 12,3 & 80,3 & 3,3 & 0,91 & 0,39 \\
\hline
\end{tabular}

\section{Confiabilidad Inter-Codificador y} Consistencia Interna de la PCNP

En la Tabla 3 consignamos los coeficientes Kappa alcanzados en cada ronda de doble-codificación para cada pregunta. Como se ve en la tabla, los coeficientes son entre buenos y excelentes para todas las preguntas, excepto para las preguntas 5 y 14 en la segunda ronda (Banerjee, Capozzoli, McSweeney \& Sinha, 1999; Landis \& Koch, 1977). La consistencia interna de la prueba fue 0,85 , evaluada con el coeficiente alfa de Cronbach. No eliminamos ninguna pregunta, pues el análisis mostró que esto reduciría la consistencia interna.

\section{Sensibilidad al Cambio Evolutivo}

La correlación $r$ de Pearson entre la edad de los participantes y su puntaje en la primera administración de la PCNP fue directa y significativa: $r(117)=0,514, p=$ 0,01 . A mayor edad los niños y niñas obtienen mayores puntajes.

En cuanto al cambio entre la primera y segunda aplicación en los niños y niñas que fueron evaluados dos veces, la prueba $t$ 
Tabla 3

Coeficientes Kappa para Dos Codificadores en Cada Pregunta de la PCNP, por Ronda de Codificación

\begin{tabular}{|c|c|c|c|c|}
\hline Pregunta & Ronda $1(n=21)$ & Ronda $2(n=21)$ & Ronda $3(n=21)$ & Total $(n=63)$ \\
\hline 1 & $1,000 * *$ & $1,000 * *$ & $0,780 * *$ & $0,935^{* *}$ \\
\hline 2 & $0,824^{* *}$ & $0,922^{* *}$ & $0,916^{* *}$ & $0,893^{* *}$ \\
\hline 3 & $1,000 * *$ & $0,554^{* *}$ & $0,578^{* *}$ & $0,732^{* *}$ \\
\hline 4 & $0,665^{* *}$ & $0,628 * *$ & $0,708 * *$ & $0,682^{* *}$ \\
\hline 5 & $0,855^{* *}$ & $0,338 * *$ & $0,845^{* *}$ & $0,692^{* *}$ \\
\hline 6 & $0,702^{* *}$ & $0,912^{* *}$ & $0,756^{* *}$ & $0,792^{* *}$ \\
\hline 7 & $0,738^{* *}$ & $0,802^{* *}$ & $0,818^{* *}$ & $0,794^{* *}$ \\
\hline 8 & $0,528^{* *}$ & $0,688^{* *}$ & $0,701^{* *}$ & $0,639 * *$ \\
\hline 9 & $0,819^{* *}$ & $0,750^{* *}$ & $0,752^{* *}$ & $0,787^{* *}$ \\
\hline 10 & $0,679^{* *}$ & $0,542^{* *}$ & $0,841^{* *}$ & $0,671^{* *}$ \\
\hline 11 & $0,525^{*}$ & $0,556^{*}$ & $0,915^{* *}$ & $0,698^{* *}$ \\
\hline 12 & $0,674^{* *}$ & $0,417^{*}$ & $0,725^{* *}$ & $0,596^{* *}$ \\
\hline 13 & $0,700 * *$ & $0,750^{* *}$ & $0,643^{* *}$ & $0,711^{* *}$ \\
\hline 14 & $0,563^{* *}$ & $0,336^{*}$ & $0,470^{*}$ & $0,490^{* *}$ \\
\hline 15 & $0,773^{* *}$ & $0,771^{* *}$ & $0,767^{* *}$ & $0,783^{* *}$ \\
\hline 16 & $0,770^{* *}$ & $0,690 * *$ & $0,483^{* *}$ & $0,655^{* *}$ \\
\hline 17 & $0,814^{* *}$ & $0,828^{* *}$ & $0,770 * *$ & $0,808^{* *}$ \\
\hline 18 & $0,444^{* *}$ & $0,597^{* *}$ & $0,699 * *$ & $0,586^{* *}$ \\
\hline 19 & $0,667^{* *}$ & $0,733^{* *}$ & $0,828^{* *}$ & $0,722^{* *}$ \\
\hline
\end{tabular}

* $\quad p<0,01$

$* * \quad p<0,001$

para muestras dependientes reveló que estos rindieron significativamente mejor en la segunda administración que en la primera $\left(M_{\text {pre }}=20,82 ; M_{\text {post }}=25,86\right), t(69)=6,18, p=$ 0,000 . Aproximadamente la mitad $(n=38)$ recibió la lectura de los tres cuentos necesarios para la prueba de CA y esto pudo haber influido en su comprensión narrativa. Un análisis de los niños y niñas que escucharon los tres cuentos y los que no, mostró que los 32 participantes que no estuvieron expuestos a las lecturas también aumentaron significativamente su puntaje, $t(31)=3,781, p$ $=0,001$, aunque menos que aquellos expuestos a las lecturas, $t(37)=5,030, p=0,000$.

\section{Relación Concurrente con Vocabulario Receptivo y Comprensión Auditiva de Cuentos}

En la Tabla 4 aparecen las correlaciones $r$ de Pearson entre PCNP, VR y CAC. Tal como se esperaba, las correlaciones de la PCNP con VR y con CAC son directas y significativas. Contrario a lo esperado, la correlación entre PCNP y VR, aunque es de menor magnitud absoluta que la de PCNP con CAC, no es significativamente diferente de esta $\left(Z_{\text {Fisher }}=0,73, p>0,05\right)$. Por otro lado, tal como se esperaba, la correlación entre PCNP y VR es significativamente menor que aquella entre CAC y VR ( $\mathrm{Z}_{\text {Fisher }}$ $=2,43, p<0,05)$, sugiriendo una influencia mayor del vocabulario en la comprensión de historias escuchadas que en la comprensión de un libro mudo.

Dado que la correlación de PCNP con VR resultó tan importante como aquella con CAC, realizamos un análisis de regresión múltiple para determinar si la CAC tiene una contribución única en los puntajes de la PCNP, más allá de la mutua relación con VR. El análisis mostró que, luego de controlar el efecto del VR, el puntaje en la CAC tuvo un efecto significativo sobre la PCNP $\left(\beta_{\text {estandarizado }}=0,332, t[69]=2,670, p=0,009\right)$, explicando un $6 \%$ adicional de la varianza de la misma. 
Tabla 4

Correlaciones $r$ de Pearson entre las Tres Variables Estudiadas

\begin{tabular}{lcc}
\hline & PCNP & CAC \\
\hline VR & $0,544^{* *}(n=117)$ & $0,688^{* *}(n=72)$ \\
PCNP & & $0,600^{* *}(n=72)$ \\
\hline
\end{tabular}

$* * p<0,001$

\section{Discusión}

Los resultados presentados sugieren que la PCNP es un instrumento apropiado para medir comprensión narrativa en preescolares chilenos de 3 y medio a 5 años. Una ventaja de esta prueba es que no requiere la lectura previa de un cuento, como las pruebas tradicionales de CAC. Para una educadora preescolar es conveniente disponer de un instrumento que se aplica en una sola sesión, en lugar de tener que exponer a los niños y niñas a un cuento y luego esperar a una nueva sesión para evaluar su comprensión, ya que existen múltiples factores que pueden interferir entre la primera lectura y la evaluación. Esto mismo se aplica al uso del instrumento en trabajos de investigación, donde es importante reducir al mínimo la intrusión de variables que afectan la validez de las evaluaciones en niños y niñas pequeños, tales como el ausentismo, la timidez y la memoria auditiva.

La capacitación para administrar la prueba resultó simple y breve y los niños y niñas respondieron al instrumento con entusiasmo e interés. El acuerdo inter-codificador fue fácil de lograr: este fue alto desde la primera ronda de codificación luego de una capacitación de dos horas. Esto sugiere que probablemente sea suficiente con un manual para que profesores e investigadores puedan aplicar y puntuar la prueba.

En cuanto a la validez concurrente de la PCNP, el estudio entrega evidencia que apoya su uso como una medida de la capacidad de los preescolares para estructurar una historia coherente. La regresión jerárquica mostró que la relación entre esta prueba y la CAC es directa y significativa, incluso luego de aislar la contribución del $\mathrm{VR}$, lo que sugiere que el instrumento mide comprensión de historias y no simplemente habilidad lingüística general. Asimismo, el hecho de que la relación de VR haya sido más baja con la PCNP que con la CAC apoya el uso de la PCNP como una medida de comprensión narrativa menos dependiente del vocabulario que una basada en historias específicas.

El aumento significativo entre la primera y la segunda aplicación de la PCNP sugiere que la prueba es sensible al progreso en la comprensión narrativa que ocurre con el desarrollo. Es posible que este cambio se deba a un efecto de aprendizaje o a una inestabilidad de la prueba, y no a su sensibilidad al crecimiento en la comprensión narrativa. Sin embargo, la correlación directa entre edad y PCNP es consistente con la interpretación de que la prueba detecta cambios asociados al crecimiento. Adicionalmente, el hecho de que los niños y niñas que recibieron las lecturas de los tres cuentos aumentaron su puntaje en mayor medida que aquellos que no, sugiere que el instrumento detecta cambios reales en la comprensión narrativa de los participantes.

El presente estudio representa una extensión de los trabajos de Paris y Paris (2003) en la medición de comprensión narrativa por varios aspectos: es un instrumento para niños y niñas más pequeños, está desarrollado en español y fue evaluado con participantes de NSE bajo y medio bajo.

La principal limitación de este estudio consiste en que la prueba utiliza un libro comercial. Aunque este libro es de escasa circulación, los niños podrían haber tenido contacto previo con él. Los profesionales que utilicen el instrumento deberían asegurarse que los niños no hayan sido expuestos 
previamente al libro. Otro problema de un libro comercial es el de los derechos de autor, que limitarían su potencial para ser utilizado como un instrumento de evaluación. El paso siguiente es construir una prueba similar en base a un libro especialmente diseñado, lo cual facilitaría la comercialización y difusión de dicha prueba.

En términos psicométricos, estudios futuros deberían incluir una prueba de memoria verbal para determinar si la PCNP es efectivamente más independiente de la memoria que una prueba de comprensión auditiva. Asimismo, sería iluminador incluir una prueba de habilidades de mentalización, para determinar su contribución a la comprensión narrativa. Otra variable que contribuiría a examinar la validez de un instrumento para medir comprensión narrativa es una medida de exposición previa a cuentos, ya que esta es una de las vías por las cuales se desarrolla esta habilidad. Un estudio que evalúe las propiedades de distintos cuentos mudos en lugar de uno solo podría también arrojar luces sobre la posibilidad de construir una prueba con preguntas genéricas que pueda aplicarse a distintos libros, en lugar de preguntas específicas para un solo libro. Finalmente, estudios longitudinales que incluyan una medida de comprensión lectora en segundo o tercer grado de educación primaria serían necesarios para examinar la validez predictiva del instrumento.

\section{Referencias}

Banerjee, M., Capozzoli, M., McSweeney, L. \& Sinha, D. (1999). Beyond Kappa: A review of interrater agreement measures. The Canadian Journal of Statistics, 27, 3-23.

Berman, R. A. \& Slobin, D. I. (1994). Relating events in narrative: Crosslinguistic developmental study. Hillsdale, NJ: Lawrence Erlbaum Associates.

Cain, K. (2003). Text comprehension and its relation to coherence and cohesion in children's fictional narratives. British Journal of Developmental Psychology, 21, 335-351.

Cain, K., Oakhill, J. V., Barnes, M. A. \& Bryant, P. E. (2001). Comprehension skill, inference-making ability, and their relation to knowledge. Memory \& Cognition, 29, 850-859.

Cain, K., Oakhill, J. \& Bryant, P. (2004). Children's reading comprehension ability: Concurrent prediction by working memory, verbal ability, and component skills. Journal of Educational Psychology, 96, 31-42.
Cromley, J. G. \& Azevedo, R. (2007). Testing and refining the direct and inferential mediation model of reading comprehension. Journal of Educational Psychology, 99, 311-325.

Da Coll, I. (1987). Chigüiro encuentra ayuda. Bogotá: Norma.

Dunn, L. M., Padilla, E. R., Lugo, D. E. \& Dunn, L. M. (1986). Test de Vocabulario en Imágenes Peabody: adaptación hispanoamericana. Circle Pines, MN: American Guidance Service.

Eastman, P. D. (1960). ¿Eres tú mi mamá? New York: Random House.

Echeverría, M., Herrera, M. O. \& Vega, M. (1993). Test de Vocabulario en Imágenes, TEVI ( $3^{\mathrm{a}}$ ed.). Concepción, Chile: Editorial Universidad de Concepción.

Elleman, A. M., Lindo, E. J., Morphy, P. \& Compton, D. L. (2009). The impact of vocabulary instruction on passage-level comprehension of school-age children: A meta-analysis. Journal of Research on Educational Effectiveness, 2, 1-44.

Gómez-Palacio, M., Rangel, E. \& Padilla, E. (1985). Kaufman Batería de Evaluación Intelectual. Manual de aplicación y calificación. Ciudad de México: Dirección General de Educación Especial.

Happé, F. G. E. (1994). An advanced test of theory of mind: Understanding of story characters' thoughts and feelings by able autistic, mentally handicapped, and normal children and adults. Journal of Autism and Developmental Disorders, 24, 129-154.

Kendeou, P., Lynch, J. S., van den Broek, P., Espin, C. A., White, M. J. \& Kremer, K. E. (2005). Developing successful readers: Building early comprehension skills through television viewing and listening. Early Childhood Education Journal, 33, 91-98.

Kendeou, P., Rapp, D. N. \& van den Broek, P. (2003). The influence of the reader's prior knowledge on text comprehension and learning from the text. En R. Nata (Ed.), Progress in education, Vol. 13 (pp. 189-209). New York: Nova Science Publishers.

Kim, O., Kendeou, P., van den Broek, P., White, M. J. \& Kremer, K. (2008). Cat, rat, and rugrats: Narrative comprehension in young children with Down syndrome. Journal of Developmental and Physical Disabilities, 20, 337-351.

Krahn, F. (2006). Huellas gigantes. Andalucía: Kalandraka Ediciones.

Landis, R. J. \& Koch, G. G. (1977). The measurement of observer agreement for categorical data. Biometrics, 33, 159-174.

Lee, L. L. \& Canter, S. M. (1971). Developmental sentence scoring: A clinical procedure for estimating syntactic development in children's spontaneous speech. Journal of Speech and Hearing Disorders, $36,315-339$.

Lynch, J. S. \& van den Broek, P. (2007). Understanding the glue of narrative structure: Children's onand off-line inferences about character's goals. Cognitive Development, 22, 323-340.

Lynch, J., van den Broek, P., Kremer, K., Kendeou, P., White, M. J. \& Lorch, E. (2008). The development of narrative comprehension and its relation to other early reading skills. Reading Psychology, 29, 327-365. 
Makdissi, H. \& Boisclair, A. (2006). Interacting reading: A context for expanding the expression of causal relations in preschoolers. Written Language \& Literacy, 9, 177-211.

Mayer, M. (1994). Frog, where are you? London: Puffin.

Ouellette, G. P. (2006). What's meaning got to do with it: The role of vocabulary in word reading and reading comprehension. Journal of Educational Psychology, 98, 554-566.

Paris, A. H. \& Paris, S. G. (2001). Children's comprehension of narrative picture books (Technical Report 3-025). Ann Arbor, MI: University of Michigan, Center for the Improvement of Early Reading Achievement.

Paris, A. H. \& Paris, S. G. (2003). Assessing narrative comprehension in young children. Reading Research Quarterly, 38, 36-76.

Peterson, R. L., Pennington, B. F., Shriberg, L. D. \& Boada, R. (2009). What influences literacy outcome in children with speech sound disorder? Journal of Speech, Language, and Hearing Research, 52, 1175-1188.

Peterson, C. \& McCabe, A. (2004). Echoing our parents: Parental influences on children's narration. En M. W. Pratt \& B. H. Fiese (Eds.), Family stories and the life course: Across time and generations (pp. 27-54). Mahwah, NJ: Lawrence Erlbaum Associates.

Raitano, N. A., Pennington, B. F., Tunick, R. A., Boada, R. \& Shriberg, L. D. (2004). Pre-literacy skills of subgroups of children with speech sound disorders. The Journal of Child Psychology and Psychiatry, 45, 821-835.

Snow, C. E., Griffin, P. \& Burns, M. S. (2005). Knowledge to support the teaching of reading: Preparing teachers for a changing world. San Francisco, CA: John Wiley \& Sons.

Stahl, S. A. \& Fairbanks, M. M. (1986). The effects of vocabulary instruction: A model-based metaanalysis. Review of Educational Research, 56, $72-110$

Fecha de recepción: Septiembre de 2009. Fecha de aceptación: Marzo de 2010.
Strasser, K. \& Lissi, M. R. (2006). Effects of shared storybook reading on narrative comprehension: Beyond frequency. Manuscrito no publicado, Escuela de Psicología, Pontificia Universidad Católica de Chile, Santiago, Chile.

Strasser, K. \& Lissi, M. R. (2009). Home and instruction effects on emergent literacy in a sample of Chilean kindergarten children. Scientific Studies of Reading. 13, 175-204.

Strasser, K. Lissi, M. R \& Larraín, A. (2009). Estudio de las interacciones persona-tratamiento en los efectos de la lectura compartida en pre-escolares (Proyecto FONDECYT N ${ }^{\circ}$ 1080241). Manuscrito no publicado, Escuela de Psicología, Pontificia Universidad Católica de Chile, Santiago, Chile.

Teale, W. H. \& Sulzby E. (1986), Emergent literacy: Reading and writing. Norwood, NJ: Ablex Publishing Corporation.

Trabasso, T. \& Wiley, J. (2005). Goal plans of action and inferences during comprehension of narratives. Discourse Processes, 39, 129-164.

Van den Broek, P., Kendeou, P., Kremer, K., Lynch, J., Butler, J., White, M. J. et al. (2005). Assessment of comprehension abilities in young children. En S. G. Paris \& S. A. Stahl (Eds.), Children reading comprehension and assessment (pp. 107-130). Mahwah, NJ: Lawrence Erlbaum Associates.

Van Kleeck, A. (2008). Providing preschool foundations for later reading comprehension: The importance of and ideas for targeting inferencing in storybook-sharing intervention. Psychology in the Schools, 45, 627-643.

Van Kleeck, A., Vander Woude, J. \& Hammett, L. (2006). Fostering literal and inferential language skills in head start preschoolers with language impairment using scripted booksharing discussions. American Journal of SpeechLanguage Pathology, 15, 85-95.

Wenner, J. (2004). Preschoolers' comprehension of goal structure in narratives. Memory, 12, 193-202.

Whitehurst, G. J. \& Lonigan, C. J. (1998). Child development and emergent literacy. Child Development, 69, 848-872. 